Research Paper:

\title{
The Process of Social Interactions in Patients With Hepatitis crosshark B Infection: A Grounded Theory Study
}

\author{
Ali Zabihi', Leila Valizadeh², Vahid Zamanzadeh ${ }^{3}$, Reza Negarandeh', Seyedeh Roghayeh Jafarian Amiri5*
}

1. Department of Nursing, School of Nursing and Midwifery, Babol University of Medical Sciences, Babol, Iran.

2. Department of Pediatric Nursing, Faculty of Nursing and Midwifery, Tabriz University of Medical Sciences, Tabriz, Iran.

3. Department of Medical-Surgical Nursing, Faculty of Nursing and Midwifery, Tabriz University of Medical Sciences, Tabriz, Iran

4. Nursing and Midwifery Care Research Center, Tehran University of Medical Sciences, Tehran, Iran.

5. Department of Nursing Management, School of Nursing and Midwifery, Babol University of Medical Sciences, Babol, Iran.

$\begin{gathered}\text { Use your device to scan } \\ \text { and read the article online }\end{gathered}$
Study. Journal of Client-Centered Nursing Care, 3(3), pp. 231-237. https://doi.org/10.32598/jccnc.3.3.231
dol: $:$ https://doi.org/10.32598/jccnc.3.3.231

Article info:

Received: 10 Dec. 2016

Accepted: 26 Mar. 2017
Keywords:

Grounded theory,

Qualitative research,

Chronic disease,

Hepatitis B, Social interaction

\begin{abstract}
A B S T RA C T
Background: The hepatitis B virus infection is a major cause of liver diseases in the world. It mostly affects psychological and social aspects as well as the mental health of patients. The aim of this study was to explain the process of social interactions in patients with Hepatitis B Virus (HBV) infection.

Methods: The present study was conducted using the grounded theory method from 2014 to 2016 in the Amol, Babol, and Tabriz health and counseling centers. The data was collected using in-depth semi-structured interviews and field notes in order to answer this question: "How does the process of social interaction occur in HBV carriers?" The participants included 18 HBV carriers, 2 spouses of the HBV carries and 2 physicians. Data analysis was conducted using Corbin and Strauss (2008).

Results: According to the data analysis, "maintenance of normal social interactions" after diagnosis of the disease is a major concern of the carriers. In order to resolve this concern, they employed some strategies, including disease concealment, protecting themselves and others, and reconciliation with the disease.

Conclusion: Following the diagnosis of hepatitis B infection, maintaining normal social interactions is considered as one of the major anxieties of the carriers. Health care teams could help them with their social interactions using the findings.
\end{abstract}

\section{Background}

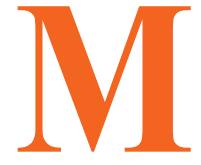

ore than 2 billion people have suffered

from hepatitis B globally, and current-

ly, $5 \%$, equal to 400 million people, carry this virus all over the world (Cecil et al. 2012). The prevalence rate of the hepatitis $B$ virus infection varies from 1.2 to $9.7 \%$ in different parts of the world (Van Damme et al. 2010; Sievert et al. 2011). In Iran, this rate is $2.14 \%$, with $2.55 \%$ in men and $2.03 \%$ in women, and

\section{* Corresponding Author:}

Seyedeh Roghayeh Jafarian Amiri, PhD

Address: Department of Nursing Management, School of Nursing and Midwifery, Babol University of Medical Sciences, Babol, Iran

Tel: +98 (911) 1157673

E-mail:jafarianamiri@gmail.com 
overall it is estimated that about 1.5 to 2.5 million people are infected by this virus (Saki et al. 2013). The most prevalent transmission methods for this disease include blood and its products, from a suffering or carrier mother to infant, unprotected sexual relations and infected syringes (Novak 2007).

Most of the hepatitis B carriers suffer from a lack of appropriate awareness and understanding of the disease, etiology, ways of disease transmission, and methods of protection and medical treatment ( $\mathrm{Ng}$ et al. 2011). Considering the fact that hepatitis is a chronic disease, it can severely affect different dimensions of patients' lives. Chronic hepatitis carriers encounter physical and mental problems, fatigue, weakness, and disappointment. A study conducted on hepatitis B carriers' attitude towards their disease revealed that the carriers feel less healthy, and since they fear transferring their disease, they face troubles in interacting with friends and relatives and are scared of communicating with them (Tran 2009).

The diagnosis of hepatitis leads to depression, anxiety, worry, frustration and disappointment in patients and they have problems in finding jobs, being able to work, and taking care of their family and the society, and sometimes even their own family will reject them (Pojoga et al. 2004; Hasanpour Dehkordi 2006; Dinstage et al. 2008). Hepatitis B carriers deal with communication problems. The main question that the researchers of the present study are looking at is how social interactions occur in hepatitis carriers. Since this concept has not been investigated formally or systematically, neither in Iran nor other parts of the world, and its different aspects are yet to be studied and well defined in the socio-cultural context of Iran, more research is required in order to present a better clarification of this concept. It is essential to conduct such a study in this field.

As a significant supplementary research method, a qualitative study often offers new and unpredictable information with more details compared to its conventional systematic quantitative counterpart (Choe et al. 2005). The grounded theory research method explores social processes underlying human interactions and their interpretation (Corbin \& Strauss 2008). Accordingly, the researchers of this study decided to apply this method in investigating the process of hepatitis B carriers' social interactions.

\section{Materials and Methods}

This study was conducted from 2014 to 2016 using the Corbin and Strauss methodology (2008). The pa- tients participated in this study were selected among those whose disease was diagnosed at least six months prior to the study, had no history of mental illness, were able to communicate, and did not have serious physical problems. Data was first generated using the purposeful sampling method; theoretical sampling was then used according to the needs of the developing theory.

Twenty-two participants were interviewed including $18 \mathrm{HBV}$ carriers, 2 spouses of carriers, and 2 physicians. The health centers of Babol, Tabriz, and the counseling center for digestive and liver diseases of Amol were selected as the locations for the interviews. The duration of each interview was 30 to 105 minutes. In order to maintain a deep connection with the data and participants' feelings, the researchers listened carefully to the interviews,transcribed them word-by-word, and coded those using MAXQDA10 immediately after the interviews. Before the interviews, participants were assured that their cooperation is voluntary and the data would be used according to confidentiality measures. Informed consent was also obtained. An ethical license for conducting the study was received with the number $5 / 4 / 49 / 29$ from the ethics committee of Tabriz University of Medical Sciences.

The interviews were conducted using questions such as: Please tell me about your experience of hepatitis B; after you were infected, how did you spend your time during the day? What measures have you been taking in response to social problems and the needs that have arisen because of the disease? As data analysis continued, some categories emerged that set the course of the next interviews; the interviews then continued based on the emerging theory. In other words, as the study proceeded, we asked questions such as: Can you give another example in this regard? What did you think when it happened? What did you do when you felt like that? Then, based on the participants' responses and the emerging categories, the interview continued using illustrative and in-depth questions such as "Could you please elaborate more on this?" "What do you mean?" and "Could you please explain more?"

Taking field notes was another technique to gather the data that was used as a complementary and corroborative method for the interview (Corbin \& Strauss 2008).Data analysis was conducted according to the analysis method proposed by Corbin and Strauss (2008) including analyzing data for concepts and the context, entering the process into the analysis, and synthesis of categories. 
In order to ensure the rigor of the data, we used the measures proposed by Guba and Lincoln (Polit \& Beck 2008). Accordingly, we tried to increase the credibility of the research through prolonged engagement, sufficient interaction with the participants, collection of valid information and member checks. In addition, we wrote our decision trail step-by-step and asked the supervisors, advisors, and experts to review the data to increase the dependability of the research. We used the faculty members' confirmation and their additional comments to increase the confirmability. Moreover, transferability was fulfilled by explaining the context in detail to provide a basis for evaluating whether it can be applied in other settings (Corbin \& Strauss 2008).

\section{Results}

A total number of 22 people participated in this study, including $18 \mathrm{HBV}$ carriers (10 males and 8 females), 2 spouses of the carriers and 2 physicians, whose age range was between 23 to 50 years and the average duration of carrying the disease was 6 years for patients. According to the participants' statements, ways of disease transmission were unknown in 9 cases $(50 \%)$, and in others it occurred from mother to infant ( 5 people, $27.8 \%$ ), via dentistry (16.7\%), and through tattooing (1 person, $5.5 \%$ ). In the present study, patients had been often diagnosed accidentally after blood donation and diagnostic tests. Since the diagnosis was unexpected for the participants, it caused some emotional reactions in them, and they experienced symptoms such as confusion, shock, anger, anxiety, and fear. On the other hand, one of the main problems of these patients in the society is issues related to social stigma, fear from patients and a significant portion of carriers' emotional reactions was related to the fact that in the future, they will deal with problems in terms of everyday social interactions with others.

One of the basic steps in data analysis was to identify the major concern and worry of HBV carriers. The underlying factors affecting the social interactions of patients were recognized with concepts such as "rejection by others, inadequate support, and the nature of the disease. "Most of the participants expressed fear from rejection and maintaining normal social interactions as their main worry; therefore, efforts for peaceful coexistence with others were defined as the central category. A participant said in this regard: I'm worried lest others may learn about my illness. I, myself, always take care. I am scared about the fact that one day someone may know it. I'm afraid they may stop their communication with me and do not visit me, and they may avoid me if they know it. (P. 15)

The following words are selected from another participant's statement about the efforts for peaceful interactions with others: I try to keep my normal communication with others and at the same time abide some rules. I observe health issues so that I may not hurt them and they won't stand away from me because of lack of observing such issues. (P. 16)

In this study, the researchers sought to identify processes that HBV carriers applied in response to the context mentioned above. The attempt for maintaining interactions was defined as the main category, while hiding the disease, taking care of oneself and others, and reconciliation with the disease were identified as subcategories (Table 1).

Table 1. Attempt to maintain interactions

\begin{tabular}{|c|c|c|}
\hline Main Theme & Sub-Themes & Primary Concepts \\
\hline & & Understating the disease \\
\hline & Disease concealment & \\
\hline & & Leading a life with secrets \\
\hline & & Controlling the Disease \\
\hline & & Vaccination \\
\hline & Protecting oneself/others & \\
\hline \multirow[t]{5}{*}{ Attempt to maintain interactions } & & Adjuvant treatment \\
\hline & & Self-protection \\
\hline & & Coping with the disease over time \\
\hline & Compromise with the disease & Religious orientations \\
\hline & & Successful counterparts \\
\hline
\end{tabular}




\section{Attempt to maintain interactions}

In order to resolve their concerns (i.e. fear of rejection, maintaining normal social interactions), the participants adopted some strategies to continue their interactions, which are described below.

\section{Disease concealment}

In most of the cases, no one other than the patient's family members knew about the person's infection. Most of the patients concealed their disease in order to maintain social interaction with others and preserve their own honor and position, as well as family and social prestige. On the other hand, the view that this disease is mostly observed among injecting drug users and profligates made the patients conceal the disease in order to avoid potential suspicions.

According to the findings, another strategy used by the participants for maintaining normal social relationships and avoiding social stigma was disease understatement. ... First I declared that I'm infected. Then I had a test and found that I don't have the disease and it's inactive and then people's reactions became better in this respect. (P. 7)

Due to the nature of HBV, the fact that disclosure of this disease influences different aspects of patients' lives and causes negative consequences on carriers and their families, lead to participants' permanent disease concealment. I try to hide my disease in the community. I even told the doctor who invited me and was talking to me not to tell these words to others, and I asked her to keep my secrets as long as possible and... (p. 3)

\section{Protecting oneself and others}

In this study, the participants used different methods and approaches to overcome their disease, cure it, and protect themselves and others. According to the findings of the present research, one of the essential measures after diagnosis of HBV was taking necessary tests and keeping track of the disease. ... I take a test almost once a year. The doctor emphasizes that I have to keep track of the experiments since this disease is a hidden one and I pay more attention to taking tests and controlling the disease. (p. 5)

One of the strategies to protect others from getting the disease immediately after diagnosis is the vaccination of the family members against the disease. After diagnosis of my disease, my husband took the test, and the result was negative and he was vaccinated three times.
My baby received both the vaccine and immunoglobulin at birth. (p. 9)

Patients and their families used other methods of treatment, which were often taking herbal medicines, in order to be able to cure the disease completely. My mother keeps track of my disease and provides me with herbal medicine and I take it. She says: "take this herbal medicine so that your disease is cured. (p. 13)

In addition to taking therapeutic actions, patients followed doctors' and health workers' advice and often implemented self-protection strategies very well. I get a lot of exercise to control my weight, I eat less fatty food, I don't smoke, I don't eat fast food, I try to consume fresh fruits and local food and keep track of my disease. (p. 17)

\section{Compromise with the disease}

Gradually, after passing the first stages of the disease, when the initial psychological reactions subside, the majority of the participants attempted to accept the disease as a parasite and part of their life. One of the most significant issues, which played a crucial role in the acceptance of and coping with the disease and the problems resulting from it, was passing of time. The disease becomes ordinary with the passing of time. Now, I have used to it and I know that I carry it. I am not ashamed of it any more. At first, I was ashamed of the disease, but now I have accepted it. (p. 10)

One of the main concepts of this study, which emerged after frequent reviews of the interviews, has been religious orientations. I have always trusted God ever since I became sick. I have always thought about God. I have always said prayers. Even my spouse wants to make sacrifice for me if I am cured. (p. 18)

Based on the findings of this study, one of the participants' approaches for accepting the disease was following other hepatitis carriers' lives and destiny. In our village, a number of other people carried hepatitis too. One or two of my relatives carry it too, for example, my aunt and her children are hepatitis carriers. Also, among my wife's relatives, her aunt and one or two of her cousins carry the disease too, and they have a normal life. Since I saw them, my disease has been normal to me and I've been more hopeful about future life... (p. 5)

\section{Discussion}

According to the findings of the present study, preserving normal social interactions was among the main 
concerns of the patients. Most of them always tried to prevent the occurrence of any disruption in their social interactions by using different strategies. Moreover, they steadily tried to release themselves from the challenges caused in their social interactions and sought tranquility under the support of a life with normal social interactions within a context emerging from inadequate support, disruption in perception and awareness, rejection, and the nature of the disease. In general, despite the existing challenges, the patients exerted strenuous efforts to peacefully coexist with others, using different strategies, while not harming them. In other studies also, patients with hepatitis were concerned about preserving normal social relations with others and made efforts to overcome this concern ( $\mathrm{Ng}$ et al. 2011; Groessl et al. 2008). In addition, like the present study, in other studies, the patients with hepatitis used various strategies to avoid harming others in order to preserve normal social interactions and peacefully coexist with them (Mohamed et al. 2012).

Patients used various processes to maintain social interactions. One such process has been disease concealment. The majority of carriers believed that this disease was more prevalent among addicts who inject drugs and sex workers; this group concealed the disease so that others would not become suspicious. The study, which was conducted in Malaysia, revealed that most patients feel ashamed of disclosing the disease to their family and caretakers. Since they are afraid of being ostracized by the community, they are obliged to hide their disease from others (Mohamed et al. 2012). The fear of negative reactions from people and losing the opportunity for forming social relationships with others led to the patients' preference for the remaining unknown (Lee et al. 2012).

One of the approaches adopted by patients for maintaining social interactions was moderating the disease during their interactions with others by selecting other titles for their illness, including silent disease, inactive, dead virus, and blood disorder. In another study, using titles like a hidden or invisible virus, carriers called their disease a silent one, did not deem that they were patients, and believed that they do no harm to other people (Lee et al. 2010).

In the present study, according to doctors' advice, one of the significant measures has been protective and therapeutic activities. Patients kept track of treatment procedures of their disease by taking periodic tests. Apart from the present research, other studies also indicated that carriers put in a lot of effort to treat and keep track of the disease after disease diagnosis (Wallace et al. 2011; Lee et al. 2010; Yang et al. 2015). In this study, another method for protecting others was vaccination of family members and immunoglobulin injection to infants whose mothers were HBV carriers. In other studies also, vaccination has been considered as one of the most crucial actions for preventing hepatitis B (Wallace et al. 2011; Cotler et al. 2011).

Since patients' routine tracking and therapeutic actions for curing and removing the disease from their lives lead to very few cases whose HBsAg test results were negative. They adopted other treatment methods, which mainly included taking herbal medicine in order to treat their disease. There are also other studies that indicate that hepatitis B carriers have taken herbal medicines (Lee et al. 2010).

Apart from medical advice, participants took selfprotective actions such as following an appropriate diet, avoiding industrial food and Acetaminophen, exercise, and walking in order to eliminate HBV from their body. The results of other studies have also revealed that hepatitis carriers had taken some actions to protect themselves and prevent disease progression (Stewart et al. 2012; Mohammadi et al. 2013). With respect to this research and other studies' findings, self-care strategy has been one of the most important protective strategies in HBV carriers and is influenced by several factors including the socioeconomic status of families and support from organizations. As time passed, patients could handle new situations and cope with the disease more easily. The results of other studies have also suggested that carriers gradually accept their disease over time (Edwards et al. 2007; Mohammadpour et al. 2012). Therefore, by considering this issue and supporting patients along this path, health care personnel can provide them with the opportunity to accept their disease over time.

Religious beliefs and having a good relationship with God assists patients very much in coping with disease, especially those like hepatitis B, which affect different dimensions of life, including physical, psychological and social aspects. Other studies' results, which were much the same as the present study's findings, indicated that patients feel less lonely or depressed, and are less inclined to morbid thoughts via thinking about God, and having respect for spiritual values and beliefs, and with this belief that everything depends upon God's will accept new conditions better (Hassanpour Dehkordi et al. 2012; Mohammad-Pour 2010). It is important to note that some of the participants of this study believed that carrying a disease depends on God's will and if God wants, one will get sick. The healthcare staff has to be aware of this and not let such beliefs and viewpoints to lead the patients to neglect healthy behavior, since this 
can spread the disease. In addition, in order to prevent the spread of disease, these staffs have to be aware of opportunists who abuse such beliefs. Therefore, religious leaders and health care teams have to consider the abuse of people's beliefs as a social challenge in order to inhibit misuse by opportunists.

After that, participants gathered information about other patients' normal life conditions, their spirits were lifted and they became more hopeful about leading a healthy and peaceful life. The results of other studies have also revealed that those HIV carriers who communicated with other patients and attended scheduled training, relaxation and stress, and anxiety reduction classes together with other counterparts, emphasized such classes' positive results and expressed that they lead to their peaceful coexistence with the disease and improved their life conditions (Mohammad-pour 2010; McCain et al. 2003; Cruess et al. 2000). Immediately after disease diagnosis, patients using different processes, attempted to prevent any disturbance in such interactions and escape incessantly from emerging challenges.

During this procedure, patients gradually learn how to continue their normal social life while suffering from this chronic disease and its resulting limitations. The majority of patients gradually became able to escape from isolation, achieve maximum possible health, keep their normal social interactions despite the presence of restrictions via a positive attitude towards themselves, others, and their own life, and also reach peaceful coexistence. Patients, people, and professional personnel have little information about the hepatitis disease. Therefore, one of the significant issues that the authorities have to prioritize is reforming health care systems and creating appropriate conditions so that patients enter health centers without feeling any fear or anxiety. Monitoring public and private health centers and handing out severe punishments to those who discriminate against such patients, do not provide them with services or delay servicing is an essential issue, which the authorities seriously have to take account of. The results of such studies can warn health authorities not only about understanding patients' experiences but also about becoming aware of their supportive role so that they can meet multiple needs of patients. In particular, regarding social interactions via designing consistent training and counseling programs and effective interventions, and assist them along the meandrous path of tackling the disease and maintaining their normal social interactions.

\section{Acknowledgments}

We would like to thank the Research and Technology Deputy of Tabriz University of Medical Sciences for their financial support of this research project. We also thank the authorities and staff at the healthcare centers of Babol, Amol, and Tabriz and especially the participants for their sincere cooperation. Corresponding Author: Seyedeh Roghayeh Jafarian Amiri, Department of Nursing, School of Medicine, Babol University of Medical Sciences.

\section{Conflict of Interest}

The authors declare no conflicts of interest.

\section{References}

Cecil, R. L., Goldman, L. \& Schafer, A. L. 2012. Goldman's Cecil medicine. Philadeelphia: Elsevier Saunders.

Choe, J. H. et al., 2005. Hepatitis B and liver cancer beliefs among Korean immigrants in Western Washington. Cancer, 104(S12), pp. 2955-8. [DOI:10.1002/cncr.21518]

Corbin, J. \& Strauss, A., 2008. Basics of qualitative research: Techniques and procedures for developing grounded theory. Los Angeles: Sage Publications. [DOI:10.4135/9781452230153]

Cotler, S. J. et al., 2011. Characterizing hepatitis B stigma in Chinese immigrants. Journal of Viral Hepatitis, 19(2), pp. 147-52. [DOI:10.1111/j.1365-2893.2011.01462.x]

Cruess, D. G. et al., 2000. Cognitive-behavioral stress management increases free testosterone and decreases psychological distress in HIV-seropositive men. Health Psychology, 19(1), pp. 12-20. [DOI:10.1037/0278-6133.19.1.12]

Dinstage, J. L., Braunwald, E., Fauci, A. S. \& Kasper, D. L., 2008. Harrison's principles of internal medicine, New York: Mc Graw-Hill.

Ebrahimi, D. N. et al., 2008. Prevalence of psychiatric disorders in hepatitis B virus carriers in Iranian charity for hepatic patients support (December 2004-August 2005). Hepatitis Monthly Summer, 8(3), pp. 201-5.

Edwards, C. R., Thompson, A. R. \& Blair, A., 2007. An overwhelming illness. Journal of Health Psychology, 12(2), pp. 203 14. [DOI:10.1177/1359105307071747]

Groessl, E. J. et al., 2008. Living with hepatitis C: Qualitative interviews with hepatitis C-infected veterans. Journal of General Internal Medicine, 23(12), pp. 1959-65. [DOI:10.1007/s11606008-0790-y]

Hasanpour Dehkordi, A., 2006. Factors affecting quality of life in cancer patients under chemotherapy in Tehran (2004). Journal of Kermanshah University of Medical Sciences, 10(2), pp. 110-9.

Hassanpour Dehkordi, A., 2012. [The process of self-care in patients with chronic hepatitis: Redesigning Orem's self-care 
theory (Persian)] [PhD dissertation]. Tehran: Tehran University of Medical Sciences.

Lee, H. et al., 2010. Complexity and uncertainty of living with an invisible virus of hepatitis B in Korea. Journal of Cancer Education, 25(3), pp. 337-42. [DOI:10.1007/s13187-010-0047-4]

Lee, H. et al., 2012. Correlates of hepatitis B virus health-related behaviors of Korean Americans: A situation-specific nursing theory. Journal of Nursing Scholarship, 44(4), pp. 315-22. [DOI:10.1111/j.1547-5069.2012.01468.x]

McCain, N. L. et al., 2003. Effects of stress management on PNIbased outcomes in persons with HIV disease. Research in Nursing \& Health, 26(2), pp. 102-17. [DOI:10.1002/nur.10074]

Mohamed, R. et al., 2012. Knowledge, attitudes and practices among people with chronic hepatitis B attending a hepatology clinic in Malaysia: A cross sectional study. BMC Public Health 12(1). [DOI:10.1186/1471-2458-12-601]

Mohammadi, N., Hassanpour Dehkordi, A. \& Nikbakhat Nasrabadi, A., 2013. Iranian patients with chronic hepatitis struggle to do self-care. Life Science Journal, 10(1), pp. 457-62.

Mohammad-Pour, A. 2010. [Living with HIV/AIDS: Patients' lived experience and development of self-care strategies (Persian)]. Tehran: Tehran University of Medical Sciences.

Mohammadpour, A., Parsa Yekta, Z. \& Nikbakht Nasrabadi, A., 2012. Coping with acquired immune deficiency infection A phenomenological study. Journal of Qualitative Research in Health Sciences, 1(3), pp. 170-81.

$\mathrm{Ng}$, C. J. et al., 2011. Uncovering the experiences and needs of patients with chronic hepatitis B infection at diagnosis. Asia Pacific Journal of Public Health, 25(1), pp. 32-40. [DOI:10.1177/1010539511413258]

Novak, E., 2007. Berek E Novak's gynecology. Philadelphia: Lippincott Williams \& Wilkins.

Pojoga, C. et al., 2004. Impaired health-related quality of life in Romanian patients with chronic viral hepatitis before antiviral therapy. European Journal of Gastroenterology \& Hepatology, 16(1), pp. 27-31. [DOI:10.1097/00042737-200401000-00005]

Polit, D. F. \& Beck, C. T., 2008. Nursing research: Generating and assessing evidence for nursing practice. Lippincott Williams \& Wilkins.

Saki, N. et al., 2013. [The challenges of hepatitis B in blood transfusion in Iran (Persian)]. Scientific Journal of Iranian Blood Transfusion Organization, 9(4), 463-77.

Sievert, W. et al., 2011. A systematic review of hepatitis C virus epidemiology in Asia, Australia and Egypt. Liver International, 31, pp. 61-80. [DOI:10.1111/j.1478-3231.2011.02540.x]

Stewart, B. J. et al., 2012. Help-seeking and coping with the psychosocial burden of chronic hepatitis C: A qualitative study of patient, hepatologist, and counsellor perspectives. International Journal of Nursing Studies, 49(5), pp. 560-9. [DOI:10.1016/j. ijnurstu.2011.11.004]

Tan, N. C., Cheah, S. L., Teo, E. K. \& Kwok, V., 2005. A qualitative study of hepatitis B carriers' understanding of their chronic infection. Asia Pacific Family Medidcine, 4(3-4), pp. 1-6.
Tran, T. T., 2009. Understanding cultural barriers in hepatitis B virus infection. Cleveland Clinic Journal of Medicine, 76(Suppl_3), pp. S10-3. [DOI:10.3949/ccjm.76.s3.03]

Van Damme, P. et al., 2010. Five years follow-up following two or three doses of a hepatitis B vaccine in adolescents aged 1115 years: A randomised controlled study. BMC Infectious Diseases, 10(1). [DOI:10.1186/1471-2334-10-357]

Wallace, J. et al., 2011. Managing chronic hepatitis B: A qualitative study exploring the perspectives of people living with chronic hepatitis B in Australia. BMC Research Notes, 4(1), p. 45. [DOI:10.1186/1756-0500-4-45]

Yang, Y. et al., 2015. Prevalence of hepatitis B and knowledge about hepatitis B among migrant workers in Shandong Province, China: A cross-sectional study. Iranian Red Crescent Medical Journal, 17(4). [DOI:10.5812/ircmj.17(4)2015.26725] 